\title{
Structural biology in the fight against COVID-19
}

\author{
How can structural biology help us understand and combat SARS-CoV-2? Researchers in the field share their \\ experiences and opinions and point to the challenges that lie ahead.
}

S ince the release of the SARS-CoV-2 genomic sequence in January 2020, structural biologists have been hard at work to reach a molecular and atomic understanding of this insidious virus. Despite disruptions and restrictions due to pandemic lockdowns, they have generated a high volume of data and scientific articles. They have also put considerable effort into peer reviewing manuscripts and project proposals on the topic. Nevertheless, several of those researchers have carved time out of their busy schedules to contribute to this Feature and tell us about the role of structural biology in the fight against SARS-CoV-2 and other emerging viral diseases, the technological developments that have allowed such rapid progress, and future directions for the field.

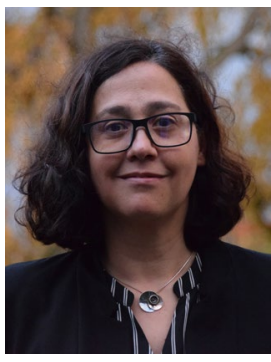

\section{Montserrat} Bárcena

Elucidating the structure and function of viral proteins is essential to understand the mechanisms of viral replication and pathogenesis and, therefore, to

pave the way for new antiviral strategies. In the last five years, cryo-EM has become a fundamental pillar of structural virology thanks to improvements in detectors and automation. The many SARS-CoV2-related structures solved by cryo-EM and single-particle analysis during the ongoing pandemic illustrate the maturity of cryo-EM as a structural technique for suspensions of molecules.

Viral infection, however, is inextricably linked to the host cell. That is where viruses use their full range of tricks to manipulate the cell and to replicate. Bringing structural virology to this natural battlefield is the next step forward. This goal has been greatly facilitated by the recent development of focused ion beam (FIB) milling to prepare thin cellular samples suitable for cryo-tomography. Cellular cryo-tomography allows the structural analysis of viral and host players in situ. It can also unveil new viral complexes, such as the molecular pore in the coronavirus replication organelle, which we recently described ${ }^{1}$. These cryo-tomograms also provide a wealth of information about the cellular context, but new tools for data analysis, mining and sharing are required to fully explore this information. Although cellular cryo-tomography currently provides relatively low-resolution data, there is no fundamental reason why near-atomic resolutions could not be achieved if it were combined with subtomogram averaging. The current technical bottlenecks are the low-throughput sample preparation and data collection steps. During the next few years, these hurdles will surely be overcome, as they are the focus of extensive ongoing developments aimed to increase automation and reproducibility. In my view, once it reaches its full potential, cellular cryo-tomography is destined to be the next cryo-EM revolution and may critically reshape our understanding of viral infection.

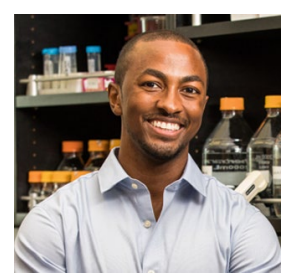

\section{Christopher O. Barnes and Pamela J. Bjorkman} In less than a year, researchers have uncovered structurefunction details

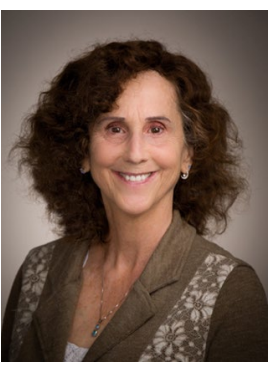
for many of the proteins encoded by SARS-CoV-2, the virus responsible for the current global pandemic. Remarkably, structures of the SARS-CoV-2 spike trimer were published in March 2020, only about two months after the viral sequence was available, aided by previous studies that established how to stabilize coronavirus spikes and the rapid turnaround time for solving structures by single-particle cryo-EM. Since then, other structures have revealed how spike binds to its angiotensin-converting enzyme 2 (ACE2) receptor, the specificities of polyclonal antibody responses in COVID19-convalescent individuals, and how monoclonal neutralizing antibodies or designed protein inhibitors bind spike to prevent infection.

Collectively, these structures provide the foundation for the development of potential therapeutics. For example, they will allow pairs of monoclonal antibodies to be chosen for treatment cocktails, they will improve antibody potencies through structure-based engineering so that they are effective at lower doses and/or are resistant to viral mutations, and they will facilitate epitope mapping to guide structure-based immunogen design. With respect to facilitating immunogen design, current candidate vaccines against SARS-CoV-2 in clinical trials did not require extensive structural biology information; however, the identification of conserved epitopes and subsequent structures of antibodies bound to spikes from SARS-CoV-2 and related coronaviruses in animal reservoirs could enable the design of immunogens to elicit cross-reactive protection against future emerging coronaviruses with pandemic potential.

A major challenge to this approach will be focusing the immune response on an epitope or epitopes that induce antibodies with neutralizing activity across multiple viral strains, an obstacle shared with HIV-1 immunogen design. With structural information and improved biochemical methods to mask distracting epitopes, a pan-neutralizing vaccine protecting against multiple coronaviruses may be attainable. Overall, structural biology is a valuable tool to gather information that will aid us in controlling the current pandemic and future outbreaks of deadly viruses.

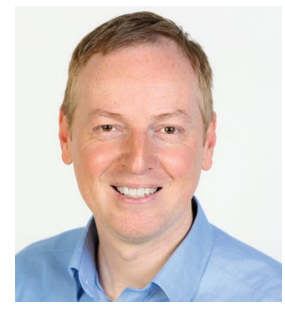

Martin Beck and Gerhard Hummer Spike, the SARS-CoV-2 surface protein, mediates host-cell entry and is the major target of neutralizing antibodies and vaccine development.

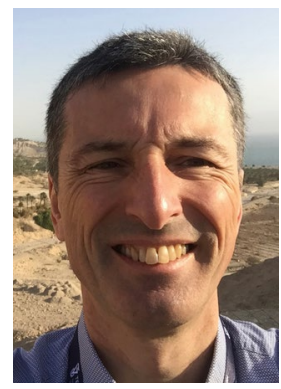
This intriguing molecular machine displays exceptional structural dynamics, both to carry out its functions and to evade the immune system. Three hinges in the long stalk between the body 
and the viral membrane render spike flexible to an extent not reported for other viral envelope proteins. The stalk itself contains an unusual right-handed coiled coil that may be metastable. Whether the hinges provide avidity to enhance cellular entry or comprise a mechanical sensor to promote conformational transitions remains unknown.

This structural plasticity extends to the receptor-binding domain (RBD), where transitions from closed to open states enable spike to bind human ACE2. Proteolytic cleavage by host proteins and low $\mathrm{pH}$ further activate spike, trigger the shedding of the S1 subunit, expose the fusion peptide and enable the dramatic conformational reorganization into the so-called post-fusion structure that drives membrane fusion and infection through a not yet fully understood mechanism.

Conformational dynamics renders spike dangerous at all stages of the infection process. Its mobile glycans shield spike from neutralizing antibodies. Dynamic clustering of spike on the viral surface combined with stalk flexibility may enhance viral avidity by allowing multiple spikes to engage with host receptors. RBD activation appears to be quasi-irreversible and can be triggered by a wide range of stimuli. Of particular concern is weak antibodies that may induce this activating transition by conformational selection, while being unable to neutralize the virus. This process may add a new twist to 'antibody-dependent enhancement,' which is normally associated with increased endocytic uptake, but here it may be amplified by nonspecific activation of spike's spring-loaded fusion machinery. Further in situ structural analysis, particularly of the actual infection events, will be important to address these questions in detail.

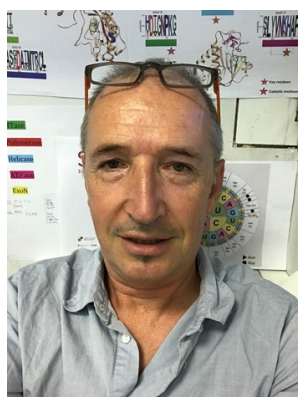

\section{Bruno Canard}

Viral diagnostics, antiviral drugs, vaccines: progress in these areas would be much slower without structural biology. Rapid developments in coronavirus $(\mathrm{CoV})$ research demonstrate this point. Before 2003, only a single $\mathrm{CoV}$ protein structure was available in the wwPDB - that of the main protease of transmissible gastroenteritis virus (TGEV). The emergence of SARS-CoV, the first highly pathogenic human $\mathrm{CoV}$, launched an intense international effort to combat the virus at the time. Modeling and molecular replacement approaches using the TGEV data yielded structural information for the SARS-CoV main protease in record time, demonstrating the power of structural biology to expedite molecular insights into emerging pathogens. The nascent era of viral structural genomics advanced and contributed, in less than a decade, to the elucidation of the majority of the structures of $\mathrm{CoV}$ proteins, making SARS-CoV one of the best understood viruses at the structural level.

The importance of high-resolution cryo-EM cannot be understated. Before 2016 crystal structures of individual $\mathrm{CoV}$ proteins and protein domains dominated. Out of 550 SARS CoV and SARS-CoV-2 PDB entries today, more than 180 were determined after 2016; $90 \%$ of these are structures of SARS-CoV-2 proteins deposited in 2020 alone. Cryo-EM accelerated considerably the pace of discovery for structures of large proteins and protein complexes, which would have been very difficult to solve using crystallography. Interestingly, overcoming future roadblocks may depend on journal editorial boards as well as on further technical advances. It is important that journals ensure reports of cryo-EM structures are accompanied by solid biochemical characterization. In addition, we need to generate functional and mechanistic data that complement the still images provided by three-dimensional (3D) structures, for example, by using the $\mathrm{X}$-ray free-electron laser and other time-resolved techniques.

Virology is of course much broader than CoV biology. A number of important human viral pathogens are waiting to be as thoroughly investigated as those of Coronaviridae. The impressive achievements made on the latter will certainly translate to the former. As next-generation DNA and RNA sequencing techniques have undergone their own technical revolution, structural and functional metagenomics of the virosphere may become the next frontier.

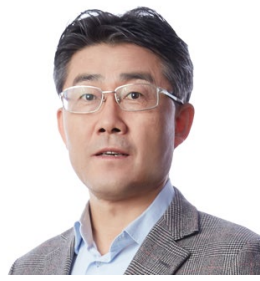
George F. Gao
The late structural biologist and crystallographer Don C. Wiley, who was my mentor at Harvard University, used to say "Get another pair of glasses to 'see' the biology by using structural biology methods." To me, that is the beauty of structural biology in contributing to our understanding of life. Trained initially as a veterinarian, I worked on animal microbiology and infectious diseases for my MSc degree and entered the field of virology and immunology during my
DPhil and postdoctoral training. I have recently become involved in public health more generally, including global health strategy and public health administration. Throughout my career, I have realized how structural biology indeed helps open our eyes so we can see biology unfolding ${ }^{2}$ and shapes the biomedical research field. Studies on antigenic peptide presentation by the major histocompatibility complex (MHC) have delineated T cell-based immunity, allowing us to clearly view and confirm hypotheses. Research on receptor-ligand interactions helps us view the host immune response in action as well as viral entry into host cells and has led to the development of small molecules and biological drugs for disease treatments. In fact, structural biology has made drug development faster than ever.

When COVID-19 emerged at the end of 2019 (ref. ${ }^{3}$ ), structural biologists immediately got to work and, using crystallography and cryo-EM, quickly defined the mechanisms of virus entry, with structures of the envelope spike protein in complex with its cellular receptor, including those from my own group, quickly published. Researchers also provided information on the main viral enzyme activities to drive inhibitor development, for example, targeting its replication machinery. In retrospect, it is clear that structural biology has played key roles in the research and development of prophylactic and therapeutic agents against many emerging and re-emerging pathogens, such as Ebola, Zika and HIV, among others.

With SARS-CoV-2, scientists and public health workers have witnessed up close the devastating toll caused by this particularly covert virus, but much remains to be learned about its biology. Though interdisciplinary efforts have increased recently, we still need more scientists who are trained in both structural and cell biology, with strong physics and biology backgrounds, as this combination of skills will enable more problem-solving-oriented research. I believe this field will be booming and will bring new research paradigm changes.

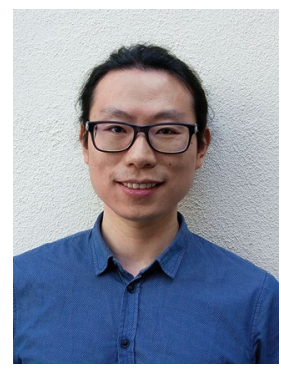

Yunyun Gao, Gianluca Santoni and Andrea Thorn As developers of structural biology methods, we usually work far from the spotlight: we are the expert users, the problem solvers, the workshop teachers and software developers, not the biologists chasing cures. When the COVID-19 

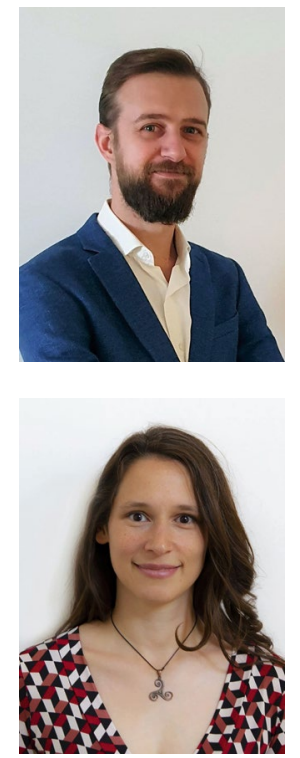

pandemic began, we asked ourselves how we could contribute to the fight against the virus and established the Coronavirus Structural Task Force $^{4}$.

\section{As early as} February 2020, we started evaluating the SARS-CoV structures available in the wwPDB and realized that many could be considerably improved. We quickly set up a website and a database containing our evaluation and revised models.

Within a week, aided by other methods developers from across the world, we began to systematically examine all published SARS-CoV structures and the new SARS-CoV- 2 structures. Soon there were 25 of us - mostly postdocs and undergraduate and graduate students - meeting online every day, working on automatic structure evaluation and revising individual structures using newly developed tools and, of course, making all of our findings available online. We also engaged in outreach activities, writing blog posts about the structural biology of SARS-CoV-2 aimed at both the scientific community and the general public, refining structures live on Twitch and offering a 3D printable virus model for schools.

We contacted the original authors of the structures that we were able to improve, which led not only to revisions of structures in the wwPDB but also to preprints being corrected before publication. Suddenly, we were being contacted by downstream users, including individual labs as well as large initiatives (such as Folding@ Home, OpenPandemics and the EU-JEDI challenge) focused on quickly finding treatments for SARS-CoV-2. The media also got wind of our work, which was covered in articles and interviews on radio and TV and in newspapers and internet outlets.

There were no tenured academics in the Task Force; we were an ad hoc collaboration of mostly junior researchers across nine time zones, brought together by a common desire to fight the pandemic. We lacked management experience, computing facilities and administrative infrastructure. Nevertheless, we were able to rapidly establish a large network of COVID19-related research, forge friendships and collaborations across national boundaries and provide improved models for multiple in silico drug discovery projects.

Following this initial burst of activity, we can now begin to assess its impact: we hope to have made a real difference in the fight against COVID-19, both scientifically and in improving the public's understanding of the virus and of scientists' efforts. We also hope that our efforts will be recognized by our peers and senior colleagues and that our decision to make all of our work rapidly and freely available will not be detrimental to our careers, since high-impact papers are the usual metric by which scientists are evaluated. We hope that the Coronavirus Structural Task Force will be a useful model for rapid, collaborative responses to pressing societal challenges.

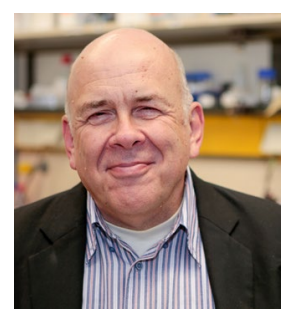

Credit: Thomas Berg

\section{Rolf Hilgenfeld}

Structural biology analyses of coronaviruses did not begin with the current SARS-CoV-2 pandemic but started in 2002 and 2003, when we reported the first crystal structures of coronavirus family

proteins, namely, the main proteases $\left(\mathrm{M}^{\mathrm{pro}}\right)$ of transmissible gastroenteritis virus (TGEV) and human coronavirus 229 $\mathrm{E}^{5,6}$. Since then, 3D structures of the spike protein, the nucleocapsid and most of the 16 non-structural proteins of SARS-CoV and other coronaviruses have been determined. Some of those structures provided important clues about the function of the non-structural proteins. Because of the high sequence similarity between SARS-CoV and SARS-CoV-2 proteins, researchers were able to rely on a solid body of structural data when SARS-CoV-2, the new coronavirus causing the current COVID-19 pandemic, emerged in early 2020.

In addition to providing insights into the function of coronaviral proteins, structures are important for the design of antiviral therapeutics. One of the most prominent targets for such compounds is coronaviral $\mathrm{M}^{\text {pro }}$. From the start, we used our early $\mathrm{M}^{\text {pro }}$ structures to design and synthesize inhibitors ${ }^{6}$. More recently, we synthesized and tested benzotriazoles and $\alpha, \beta$-unsaturated esters as $\mathrm{M}^{\text {pro }}$ blockers, followed by $\alpha$-ketoamides. Combining structural biology with medicinal chemistry in the same laboratory was a successful strategy, as almost no one will know the structure of the target better than the scientist who determined it. It gives me immense pleasure to design small-molecule inhibitors based on crystal structures determined by my group - creating a new molecule is clearly different from analyzing the structure of its target, and I encourage structural biologists to integrate medicinal chemistry into their efforts.

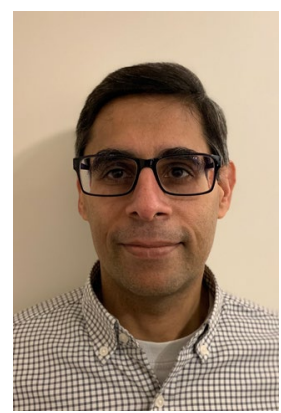

Ardan Patwardhan The proliferation of cryo-EM studies in structural biology has led to concomitant increases in depositions to the structural archives the EMDB (for 3D volumes), EMPIAR (for image data) and the PDB (for atomic models). The EMDB reached a milestone in February 2020 with its 10,000th released entry. We anticipated a slowdown due to the pandemic shutdowns but, to the contrary, depositions have continued at pace: as of 10 November 2020, there were 213 EMDB entries related to SARS-CoV-2 from 70 publications and 12 EMPIAR entries from 9 publications. The viral spike protein has the highest number of entries (168 in the EMDB), followed by the RNA-dependent RNA polymerase, although the diversity of sample types has grown over time.

There is a need for improved validation of cryo-EM maps and models and for improvements to and expansion of the data and metadata collected to enable validation. We also need to be able to effectively deal with the increasing complexity of cryo-EM experiments, which result in equally complex multimap and multimodel depositions. All of these challenges have been highlighted by community experts as requiring urgent attention, for example, at the Wellcome Trust UK EM Validation Network (WT-EM-VALNET) "Frontiers in cryo-EM validation" (January 2019) and the wwPDB single-particle EM data management (January 2020) workshops. With the added urgency of the pandemic and the pressure to rapidly publish and make SARS-CoV-2 structures available to the public, it is perhaps understandable that there may be a greater preponderance of issues with those entries (for example, modeling errors or maps and models that are described in a paper but not deposited). On the other hand, it could simply be that these structures have been more scrutinized than most; this became evident at the recent WT-EM-VALNET Zoomposium "Cryo-EM Validation in the Age of SARS-CoV-2," as many different groups (for example, the Coronavirus Structural Task Force coalition) have been carefully examining and rerefining atomic models. 
In the future, it would be useful for all of the stakeholders involved, including the organizations running the archives, journal publishers, funding agencies and the structural community, to leverage the experience from SARS-CoV-2 to improve policies and procedures for structural data archiving. For example, one idea is to ask authors to provide a table with their manuscript listing all figure parts depicting maps and/or models along with their respective accession codes. This would greatly facilitate the review process and ensure that no map or model depositions get missed, while providing greater clarity to readers wanting to delve deeper by examining the entries. Another idea is to move toward a routine deposition of some form of image data (at least the particle stacks corresponding to EMDB map depositions) to EMPIAR to enable recalculation of the map.
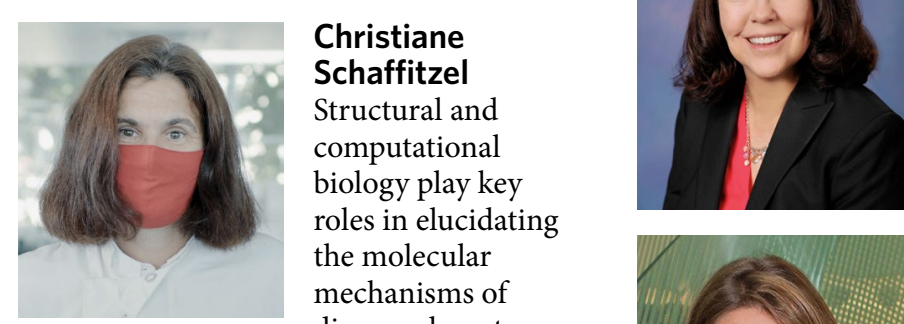

biology play key roles in elucidating the molecular mechanisms of disease phenotypes

and in driving drug discovery. This is exemplified by the current fast-paced efforts to respond to the COVID-19 pandemic. To aid these endeavors, the University of Bristol set up the COVID Emergency Group (UNCOVER), coordinated by Bristol clinician Adam Finn, which initiated multidisciplinary collaborations among virologists, chemists, physicians, pharmacologists and others to accelerate our understanding of COVID-19 and to design tailored treatments urgently needed to overcome the crisis. In our work, we discovered a druggable pocket in the SARS-CoV-2 spike glycoprotein, the viral component that mediates the interaction with ACE2 and that is essential for infection ${ }^{7}$. In our cryo-EM structure of spike, this pocket was occupied by linoleic acid (LA), an essential polyunsaturated free fatty acid (FFA). LA stabilized spike in a locked conformation that is incompatible with ACE2 binding and is thus considered 'non-infective.' Intriguingly, LA synergizes with remdesivir, the first drug approved for COVID-19 treatment, to block viral replication in cells?.

Our discovery has actionable implications: the data suggest that LA could act as a prophylactic antiviral. Intriguingly, sera from patients with COVID-19 have decreased levels of FFAs, including LA $^{8}$; therefore, LA supplementation may be beneficial. Small molecules binding the FFA-binding pocket (LA mimics) have potential as future antivirals, as they may lock spike in a non-infective conformation. Finally, the body metabolizes LA to eicosanoids, including prostaglandin, which are key molecules in immune modulation. In addition, LA-based phospholipids maintain the fluidity of cell membranes and surface tension in the lung. While more research is required to decipher the interplay of FFAs, SARS-CoV-2 and COVID-19 pathology, targeting the LA metabolic axis could help prevent rampant inflammatory responses in severe cases of COVID-19 and reduce respiratory distress and the risk of pneumonia in patients.
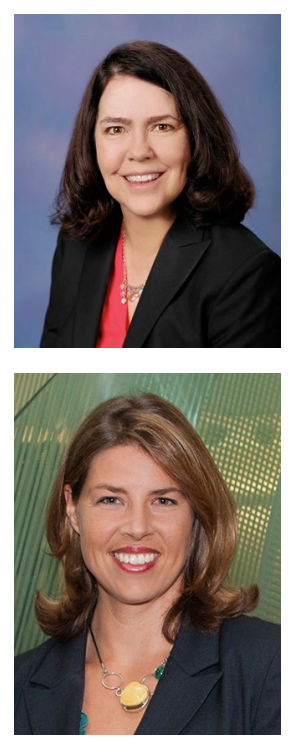

\section{Sharon L. Schendel and Erica Ollmann Saphire}

At no time in history have we been better able to image our viral enemies and launch immune defenses than now. The availability of direct electron detection, coupled with advanced instrumentation for selecting and analyzing cells of interest, lays at our feet a wealth of stunning detail and paves the route forward for medical defenses. Advances in cryo-EM instrumentation and data analysis freed us from the need to screen thousands of crystals and shrank the time needed to produce structures from years to days. Moreover, we can now begin to capture the structural and temporal heterogeneity and flexibility through which actual biology is manifested. Our new ability to detect subtle differences in glycosylation and antibody occupancy can further guide therapeutic development. We can precisely analyze structures of uniform monoclonal antibodies in a delivered therapeutic as well as the breadth of antibodies in the polyclonal milieu of actual patients.

We are harnessing these new capabilities in collaborative efforts to characterize the features of antibodies that best correlate with the ability to protect living things. La Jolla Institute for Immunology (LJI) is the headquarters for two global consortia to discover antibody-based therapeutics: one group is focused on SARS-CoV-2

and the other on re-emerging threats like Ebola, Lassa and chikungunya. Dozens of contributors provided antibodies against these viruses to be analyzed side-by-side to reveal multiple parallel facets of antibody function. We will pair results from these analyses with structural studies to define, in exquisite detail, how antibodies act to provide protection against deadly viral infections. Together, the speed and illumination of our instrumentation and the biophysical, biochemical and immunological information gained through collaboration fill gaps in knowledge and allow us to claim new victories in the fight against infectious disease

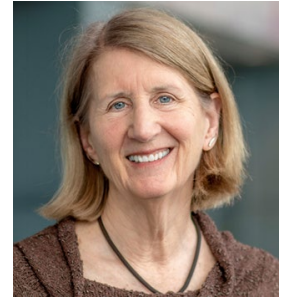

Credit: Stephanie King

\section{Janet Smith}

Three-dimensional structures of macromolecules are the basis for much of our understanding of the molecular aspects of pathogenesis. With structures, we can explain pathogen resistance

to antibiotic and antiviral drugs, the transfer of proteins and DNA into foreign cells, and the membrane fusion events that lead to infection by viruses such as influenza, HIV-1 and SARS-CoV-2. Structural biology has also greatly advanced our knowledge of the molecular pathways of the innate and adaptive immune systems. Ribosome structural biology is being used to develop new antibiotics, and RNA structures, together with an atomic-level understanding of translation, have led to the development of new RNA-based vaccines. Our lab's structural studies of the flavivirus NS1 protein fostered a new understanding of the function of this enigmatic virulence factor. NS1 was found to have a previously unknown role in virus packaging and to induce the vascular leak that is a hallmark of severe dengue disease. The structure opened new possibilities for the development of antivirals and vaccines against dengue, Zika, West Nile and other flaviviruses.

The speed and reach of structure determination have increased due to many technological advances, for example, hot microbeams at synchrotron sources for crystallography; high-speed, low-noise detectors for electron microscopy and crystallography; and faster, cheaper computing for all methods. These and other advances put the 'biology' in structural biology, allowing structural knowledge to be prospective rather than retrospective. Nowhere is this more apparent than in the response of the research community to 
the COVID-19 pandemic. In the first ten months following the publication of the SARS-CoV-2 genome sequence, the PDB released more than 560 structures of viral proteins and RNA from researchers around the world ( $72 \%$ of the structures were from crystallography, 27\% from cryo-EM and $1 \%$ from NMR). These are a foundation for vaccine and antiviral development efforts and for new discoveries in the basic biology of both host and pathogen.

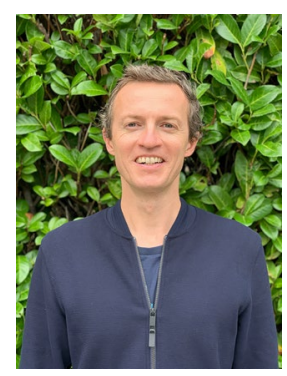

\section{David Veesler}

The recent emergence of a previously unknown coronavirus (designated SARS-CoV-2) resulted in the ongoing COVID-19 pandemic, which has claimed over 1.2 million lives and brought the world to a standstill. On 10 January 2020, the first genome sequence of a SARS-CoV-2 isolate was made publicly available and initiated the race to understand the molecular architecture and functional intricacies of this new pathogen. Cryo-EM structures of the SARS-CoV-2 spike glycoprotein and of the RNA-dependent RNA polymerase, along with crystal structures of the spike receptor-binding domain in complex with its host receptor, ACE2, were determined in just a few weeks. These results provided blueprints of the viral infection and replication machineries, which have been used by thousands of researchers worldwide for the design of vaccines and viral inhibitors. Structures of the spike glycoprotein ectodomain revealed the conformational dynamics of the receptor-binding domain, alternatively exposing and masking the ACE2-interaction site in a manner reminiscent of what had been previously observed for SARS-CoV and MERS-CoV and was postulated to contribute to immune evasion. Cryo-ET studies of fixed SARS-CoV-2 revealed the marked flexibility of full-length spike trimers relative to the viral membrane, along with the supramolecular organization of this pleiomorphic virus.

A wave of spike glycoprotein structures in complex with antibodies yielded insights into the host humoral immune response to SARS-CoV-2 infection and delineated an antigenic map of the receptor-binding domain, which accounts for most of the neutralizing activity in COVID-19 convalescent plasma. Past and present single-particle cryo-EM studies have enabled the engineering of spike trimers with altered conformation and enhanced stability and informed the rational design of the unprecedentedly large number of protein subunit, nucleic acid and viral vector vaccines currently in development. Finally, structural biology has supported the development and uncovered the mechanism of action of computationally designed ultrapotent miniprotein inhibitors of viral entry, heralding a new era for the rapid development of prophylactic and therapeutic countermeasures against emerging pathogens.

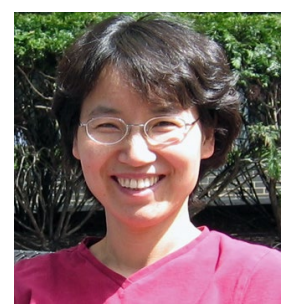
Peijun Zhang In response to the COVID-19 pandemic, efforts from scientists around the world have yielded a great deal of knowledge on the etiological agent, SARS-CoV-2. Structural understanding of the viral components is key to the development of therapeutics and, so far, work has concentrated on viral spikes, the major protease, RNA polymerase and other non-structural proteins, as well as interactions between spike and ACE2 and neutralizing antibodies. Researchers have used a range of tools, from synchrotron-based X-ray crystallography to cryo-EM and cryo-ET. The last two techniques are essential for in vitro and in situ study to obtain information in the context of a virion. All of these techniques will be important in tackling the challenges that lie ahead - including the structural elucidation of the remaining viral components, such as $\mathrm{N}, \mathrm{E}$, and $\mathrm{M}$ proteins and ribonucleoproteins, which are small, flexible and heterogeneous, and in some cases membrane bound - and they will be pivotal in understanding the entire virus architecture and its genome organization.

Much less is known about the SARS-CoV-2 replicative cycle in the native cellular environment. Viral genome replication, assembly and egress is a multistage process that is critically important, as it comprises multiple targets for medical interventions to stop infection. We have recently developed a unique correlative multimodal, multiscale cryo-imaging approach, combining in cellulo soft X-ray cryo-tomography and serial cryo-focused ion beam/scanning electron microscope volume imaging of the entire near-native SARS-CoV-2-infected cell, with high-resolution cryo-ET and subtomogram averaging of individual components. This approach enables a holistic view of the infection process, from the level of the whole cell to individual molecules, revealing not only the cytopathic effects of SARS-CoV-2 infection but also new pathways of SARS-CoV-2 assembly and egress. Further development in the area of labeling individual viral and host proteins involved in the process will be essential to allow a correlative structural analysis at the molecular level.

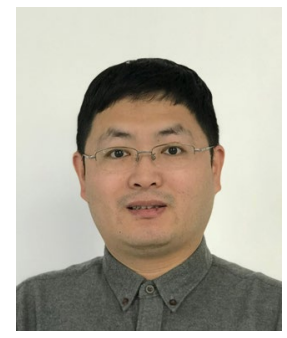

\section{Qiang Zhou}

SARS-CoV-2 is the seventh coronavirus known to infect humans and has caused the COVID19 pandemic. SARS-CoV-2 shares $80 \%$ sequence identity with

SARS-CoV, responsible for the 2002-2003 SARS epidemic. Both coronaviruses use ACE2 to invade cells and cause similar symptoms, but SARS-CoV-2 has much higher infectivity.

Structural biology has an essential role in antiviral research for SARS-CoV-2. Structures for almost all of the important antiviral targets, including the spike protein, the main protease and the RNA-dependent RNA polymerase, have been determined. Those studies have benefited from the 'resolution revolution' in single-particle cryo-EM, which has greatly improved the efficiency of structure determination. Within two months of the outbreak, structures of spike and its complex with ACE2 were determined, laying the foundation for subsequent studies of the invasion mechanism and for the development of antiviral drugs, neutralizing antibodies and vaccines.

The huge impact of the pandemic has driven many structural biologists to participate in SARS-CoV-2-related research as much as possible, pushing the field into new territory. At present, there are hundreds of entries in the wwPDB related to SARS-CoV-2; a large fraction involves small molecules and neutralizing antibodies that could potentially be used for antiviral treatment. The advances in cryo-ET technology have enabled direct visualization of the architecture of SARS-CoV-2 virion particles, opening the door for in situ structural biology.

Since the beginning of the 21 st century, frequent and periodic outbreaks of infectious diseases caused by viruses have become the norm. Developing specific drugs for such diseases is an urgent need and poses a great challenge. The progress of structural biology, and especially of cryo-EM technology, will empower antiviral drug development and help combat viral diseases. 
Montserrat Bárcena', Christopher O. Barnes², Martin Beck ${ }^{3}$, Pamela J. Bjorkman², Bruno Canard ${ }^{4}$, George F. Gao ${ }^{5}$, Yunyun $\mathrm{Gao}^{6}$, Rolf Hilgenfeld ${ }^{7}$, Gerhard Hummer ${ }^{3}$, Ardan Patwardhan ${ }^{8}$, Gianluca Santoni ${ }^{9}$, Erica Ollmann Saphire ${ }^{10}$, Christiane Schaffitzel ${ }^{11}$, Sharon L. Schendel ${ }^{10}$, Janet L. Smith ${ }^{12}$, Andrea Thorn ${ }^{6}$,

David Veesler ${ }^{13}$, Peijun Zhang ${ }^{14,15}$ and Qiang Zhou ${ }^{16,17}$

${ }^{1}$ Leiden University Medical Center, Leiden, the Netherlands. ${ }^{2}$ California Institute of Technology, Pasadena, CA, USA. ${ }^{3}$ Max Planck Institute of Biophysics, Frankfurt am Main, Germany. ${ }^{4}$ Centre National de la Recherche Scientifique and Aix-Marseille University, Marseille, France. ${ }^{5} \mathrm{CAS}$ Key Laboratory of Pathogen Microbiology and Immunology, Institute of Microbiology, Chinese Academy of Sciences, Beijing, China. ${ }^{6} \mathrm{HARBOR}$, University of Hamburg, Hamburg, Germany. ${ }^{7}$ Institute of Molecular Medicine, University of Lübeck,
Lübeck, Germany. ${ }^{8}$ European Molecular Biology Laboratory (EMBL-EBI) European Bioinformatics Institute, Hinxton, UK. ${ }^{9}$ European Synchrotron Radiation Facility, Grenoble, France. ${ }^{10}$ Center for Infectious Disease and Vaccine Research, La Jolla Institute for Immunology, La Jolla, CA, USA. ${ }^{11}$ School of Biochemistry and Bristol Synthetic Biology Centre (BrisSynBio), University of Bristol, Bristol, UK. ${ }^{12}$ Life Sciences Institute and Department of Biological Chemistry, University of Michigan, Ann Arbor, MI, USA. ${ }^{13}$ University of Washington, Seattle, WA, USA. ${ }^{14}$ Division of Structural Biology, Wellcome Trust Centre for Human Genetics, University of Oxford, Oxford, UK. ${ }^{15}$ Diamond Light Source, Harwell Science and Innovation Campus, Didcot, UK. ${ }^{16}$ Center for Infectious Disease Research, Westlake Laboratory of Life Sciences and Biomedicine, Key Laboratory of Structural Biology of Zhejiang Province, School of Life Sciences, Westlake University, Hangzhou, China. ${ }^{17}$ Institute of Biology, Westlake Institute for Advanced Study, Hangzhou, China.
Published online: 12 January 2021

https://doi.org/10.1038/s41594-020-00544-8

References

1. Wolff, G. et al. Science 369, 1395-1398 (2020).

2. Chen, Y., Shi, Y., Cheng, H., An, Y.-Q. \& Gao, G. F. IUBMB Life 61, 579-590 (2009).

3. Tan, W. et al. China CDC Wkly 2, 61-62 (2020).

4. Croll, T. et al. Preprint at bioRxiv https://doi org/10.1101/2020.10.07.307546 (2020).

5. Anand, K. et al. EMBO J. 21, 3213-3224 (2002).

6. Anand, K., Ziebuhr, J., Wadhwani, P., Mesters, J. R. \& Hilgenfeld, R. Science 300, 1763-1767 (2003).

7. Toelzer, C. et al. Science https://doi.org/10.1126/science.abd3255 (2020).

8. Shen, B. et al. Cell 182, 59-72.e15 (2020).

Competing interests

C.S. declares that patent applications describing methods and material compositions based on the presented observations have been filed. D.V. is a consultant for Vir Biotechnology Inc. The Veesler laboratory has received a sponsored research agreement from Vir Biotechnology Inc. All other authors declare no competing interests. 